\title{
The Long Noncoding RNA ANRIL Promotes Cell Apoptosis in Lipopolysaccharide-Induced Acute Kidney Injury Mediated by the TLR4/ Nuclear Factor-Kappa B Pathway
}

\author{
Ye Zhu $^{\text {a }}$ Sheng-Wei Wei ${ }^{b}$ Ao Ding ${ }^{a}$ Wei-Ping Zhu ${ }^{a}$ Mei-Fang Mai ${ }^{a}$ \\ Tong-Xia Cui a Hui Yang ${ }^{c}$ Hua Zhang ${ }^{c}$ \\ aDepartment of Nephrology, The Fifth Affiliated Hospital of Sun Yat-sen University, Zhuhai, \\ China; ${ }^{b}$ Department of Urology, The Fifth Affiliated Hospital of Sun Yat-sen University, \\ Zhuhai, China; ' Department of Rheumatology, The Fifth Affiliated Hospital of Sun Yat-sen \\ University, Zhuhai, China
}

\section{Keywords}

Endotoxin · Acute kidney injury · ANRIL · miR-199a · Nuclear factor-kappa B

\begin{abstract}
Background/Aims: The purpose of this study is to analyze the expression and biological function of IncRNA ANRIL, microRNA-199a, TLR4, and nuclear factor-kappa B (NF-kB) in acute renal injury (AKI) induced by lipopolysaccharide (LPS). Methods: The levels of ANRIL and microRNA-199a in mouse cells and kidneys were detected by quantitative-polymerase chain reaction. Western blot analysis was used for the NF-KB pathway protein. MTT assay was used for cell viability. Enzyme-linked immunosorbent assay was used for the secretion of inflammatory factors in mouse kidney tissue. Apoptosis was measured by flow cytometry and Western blotting. The potential binding region between ANRIL and miR-199a was verified by luciferase reporter assay. Results: The upregulation of ANRIL can reduce the expression of microRNA-199a and increases the number of apoptotic cells. The expression levels of ANRIL in LPS-induced AKI mice and LPS-treated HK2 cells were upregulated compared with the control group. Overexpression of ANRIL increased apoptosis and promoted TLR4 (Toll-like receptor 4), NF-KB phosphorylation, and downstream transcription factor production. Conclusion: ANRIL/NF-KB pathway in LPS-induced apoptosis provided theoretical guidance for ANRIL in the treatment of AKI.

Y.Z. and S.-W.W. contributed equally to this work. 


\section{Kidney \\ Blood Pressure \\ Research}

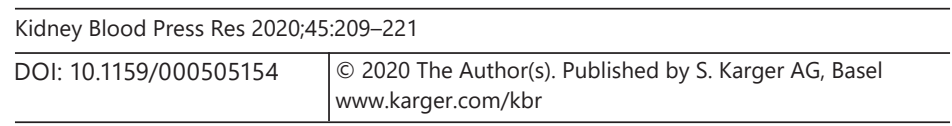

Zhu et al.: Long Noncoding RNA ANRIL and Kidney Injury

\section{Introduction}

The pathogenesis of sepsis is a systemic inflammatory response that is uncontrollable [1, $2]$. The study found that the kidney is a particularly vulnerable organ throughout the development of sepsis [3]. Acute renal injury (AKI) is a common complication. Kidney damage is a high incidence of sepsis complications and an independent risk factor for mortality [4, 5]. The study found that about half of patients with sepsis will suffer from damage to their kidneys [6]. The mortality rate of acute kidney injury is over $70 \%$, especially for patients who need dialysis treatment. Sepsis is the most important cause of acute kidney injury. Its pathophysiology is complex and not fully elucidated [7].

Recent studies have further recognized the occurrence of AKI, including renal microcirculatory disorders, glomerular circulation, inflammatory markers, and cell death $[8,9]$. In this pathogenesis, cascading amplified inflammatory responses play a pivotal role in sepsis AKI [10]. The latest research shows that both miRNA and IncRNA are involved in the process of immune regulation $[11,12]$. But little research has been done on sepsis. Therefore, the upstream regulatory signals of inflammatory factors release may be a new treatment direction for sepsis.

LncRNA participates in protein-coding genes and epigenetic groups through a variety of mechanisms expression. LncRNA can cross the promoter region of downstream proteincoding genes and interfere with the binding of transcription factors to DNA promoters, thus inhibiting gene expression [13]. ANRIL has an abnormal expression in a variety of diseases and has a certain influence on the development and behavior of the disease [14, 15]. ANRIL has been considered as a disease-causing gene. Nevertheless, the biological senses of ANRIL in AKI have not been fully studied and revealed.

It has been found that noncoding RNAs, especially lncRNA and miRNAs, play a vital role in the development of diseases. LncRNA and miRNA can regulate the occurrence of disease by multisite and multitarget regulation and regulate the interaction by regulating their relative abundance. Gene expression regulated by lncRNA-microRNA helps the communication and regulation between them and promotes physiological and pathological processes in diseases $[16,17]$. However, there are few studies on the mechanism of action of its lncRNAmiRNA network and their downstream target genes in AKI. Therefore, it is essential to analyze the role of IncRNA-miRNA networks and their relevance in the pathogenesis of AKI. In view of the above problems, this study intended to explore the effects of IncRNA-miRNA expression on the pathogenesis of AKI by detecting the expression of ANRIL and miRNA-199a in mice kidneys and cells and explore the effects of ANRIL on the biological behavior of HK-2 cells through the miRNA-199a-3p/PAWR axis, so as to understand the mechanism of the occurrence and development of AKI, which may provide reliable basis for the clinical diagnosis and targeted treatment of AKI.

\section{Methods and Materials}

\section{Animal Model}

Male C57BL/6 mice aged 8-10 years and weighing 25-30 g were purchased from the Animal Laboratory Center of Sichuan University in Chengdu. The researchers established the lipopolysaccharide (LPS)-induced AKI animal model by intraperitoneal injection of PS (i.p.). The control group received the same volume of the carrier (carrier group). The experimental scheme was approved by the Animal Protection and Use Ethics Committee of the Sichuan University of China. 


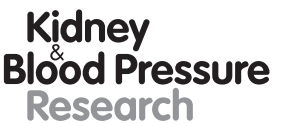

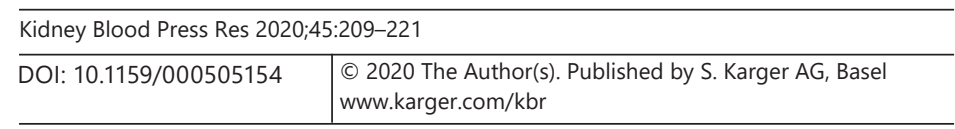

Zhu et al.: Long Noncoding RNA ANRIL and Kidney Injury

\section{Lentiviral Vector Construction and Infection}

The lentiviral vector was constructed by connecting the DNA links for human ANRIL with LV10-CMV-RFP-Puro vectors. A vector that cannot target a negative sequence of any gene was used as a negative control group. Mice were then infected with shANRIL.

\section{Renal Function Measurement}

After $6 \mathrm{~h}$ of modeling, the whole blood of mouse eyeballs was taken, centrifuged at 3,500 $r / \mathrm{min}^{-1}$ for $10 \mathrm{~min}$, and the supernatant was taken. Serum creatinine (Scr) and blood urea nitrogen (BUN) were detected by MODEMP800 biochemical analyzer.

\section{Renal Histopathology}

The right kidney tissue of the mice was taken, fixed by $40 \mathrm{~g} \cdot \mathrm{L}^{-1}$ paraformaldehyde, and dehydrated with gradient alcohol. After decalcified, embedded in paraffin, and hematoxylin and eosin staining, the morphological changes of kidney tissues were observed under a special refrigerated digital photographic device ( $\times 400$ times) for optical microscopy.

\section{TUNEL (TdT-Mediated Biotin-dUTP Nick-End Labelling) Staining}

The mouse kidney was fixed and made into paraffin sections. After dewaxing and hydration, it was sealed according to the instructions in the Tunnel staining kit, and microscope was used to observe and take pictures at the same time.

\section{The Quantitative Real-Time Polymerase Chain Reaction Analysis}

Winooski et al. used a total RNA extraction kit to isolate and extract total DNA from kidney tissues of mice, and scandlop 100 analyzer was used to measure the mRNA concentration. Quantitative real-time polymerase chain reaction was performed in the polymerase chain reaction system after reverse transcription. The expression of related genes was standardized by $\mathrm{U} 6$ and analyzed by the $2^{\text {DDCTT }}$ method.

\section{Cell Culture}

HK-2 cells were purchased from the cell bank of the Chinese Academy of Sciences and cultured in medium containing $10 \%$ fetal bovine serum. The cell culture was carried out in a cell culture chamber at $37^{\circ} \mathrm{C}$, with $5 \%$ carbon dioxide in the cell culture chamber. LPS with concentrations of $0,0.01,0.1,1$, and $10 \mathrm{~g} / \mathrm{mL}$ were used to treat hk- 2 cells for $24 \mathrm{~h}$, so as to induce the model of cellular sepsis AKI. ARIL overexpression vector was constructed and cloned into pcdna3.1 (+). Positive ANRIL pcDNA plasmid was identified by sequencing. According to the manufacturer's instructions, when the cells grew to $70 \%$, the lps-induced AKI model was briefly transfected with Anril-cDNA plasmid, and then transfected with Lipofectamine 2000. The above hk-2 cells were treated with an nuclear factor-kappa B (NF-B) inhibitor.

\section{Enzyme-Linked Immunosorbent Assay}

The blood of the mice prepared in the above procedure was centrifuged at room temperature 3,000 r/min for $10 \mathrm{~min}$ to obtain serum samples, and the urea nitrogen and creatinine were detected using a diagnostic kit and an automatic biochemical analyzer. The concentrations of interleukin (IL)-1 $\beta$, IL-6, and tumor necrosis factor alpha (TNF- $\alpha$ ) in serum were quantified in strict accordance with the procedures of the enzyme-linked immunosorbent assay kit. 
A.

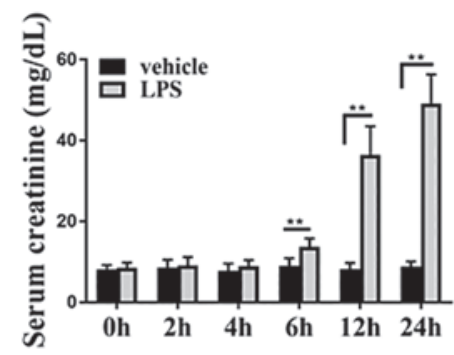

D.

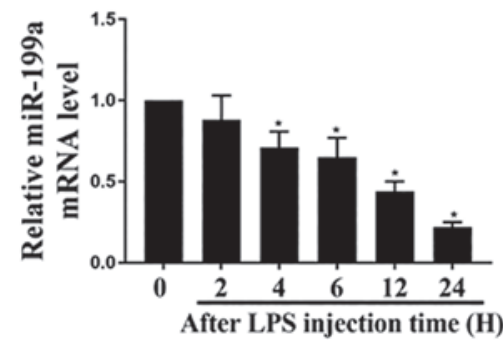

B.

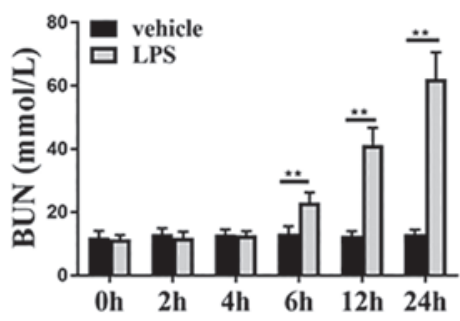

F.

E.

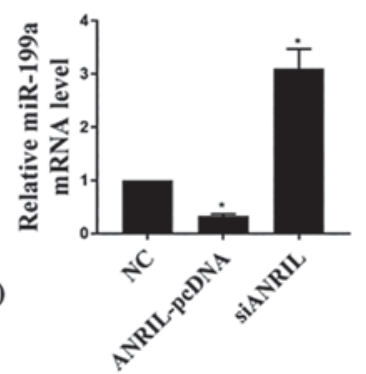

G.
C.

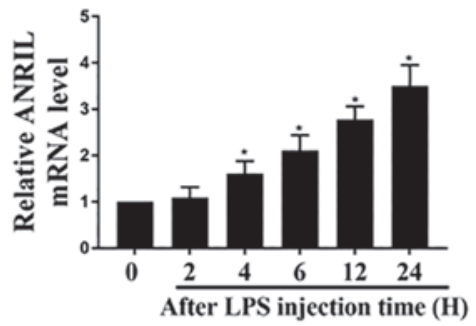

ANRIL mutant $\quad 5^{\prime}$... tgctctgttccagcctacaaatc....3

ANRIL wild type $\quad 5^{\prime}$ '...tgctctgttccagccACACTGGC....3 miR-199a $33^{\prime}$-cttgtccatcagactTGTGACCC-5

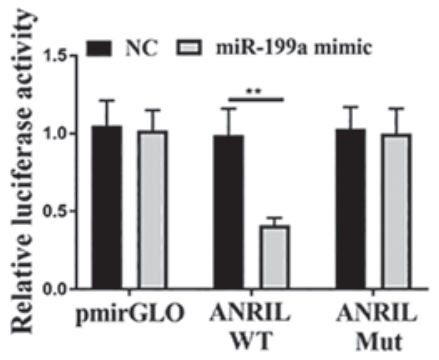

Fig. 1. Increased expression of ANRIL in LPS-induced renal injury in vivo. A Scr levels. B BUN levels. C The expression of ANRIL in LPS-induced AKI kidneys. $n=6,{ }^{*} p<0.05$. D The expression of miR-199a mRNA levels in AKI kidneys. E The eMiR-199a expression in HK-2 cells. F Complementary binding sites with mir-199a and 3-utr. G Luciferase reporter assay. Scr, serum creatinine; LPS, lipopolysaccharide; BUN, blood urea nitrogen, ${ }^{* *} p<0.05$.

\section{Luciferase Reporter Gene Assay}

The 3'-UTR of the ANRIEL cDNA was amplified by a polymerase chain reaction and cloned downstream of the firefly luciferase gene pGL3. The pGL3-luc-ANRIL wild type/mutant was introduced into HEK 293T cells with mir-199a using Lipofectin 2000. Luciferase activity was measured using a dual-luciferase reporter kit after 1 day of transfection. The activity of firefly and breviscapine luciferase was determined using a dual-luciferase reporter system, normalized to Renilla luciferase activity.

\section{MTT Determination}

HEK 293T cells were inoculated into 96-well plate, the density of cell inoculation was $1 \times 10,000$ cells/hole. After inoculation, HEK $293 \mathrm{~T}$ cells were cultured for $24 \mathrm{~h}$. Thereafter, MTT of $10 \mathrm{mU} / \mathrm{L} 5 \mathrm{mg} / \mathrm{mL}$ was added into the cell pore and incubated for $4 \mathrm{~h}$. After incubation, $100 \mathrm{mU} / \mathrm{L}$ dimethyl sulfoxide was added. The absorbance of each cell pore at $570 \mathrm{~nm}$ was measured by an enzyme label.

\section{Western Blot}

Protein was isolated from HK-2 cells or renal tissue using RIPA solution. The lysate was applied to $12 \%$ polyacrylamide gel electrophoresis and transferred to polyvinylidene fluoride membrane. The membrane was blocked by 5\% milk and combined with nf-b p65 (Abeam, 


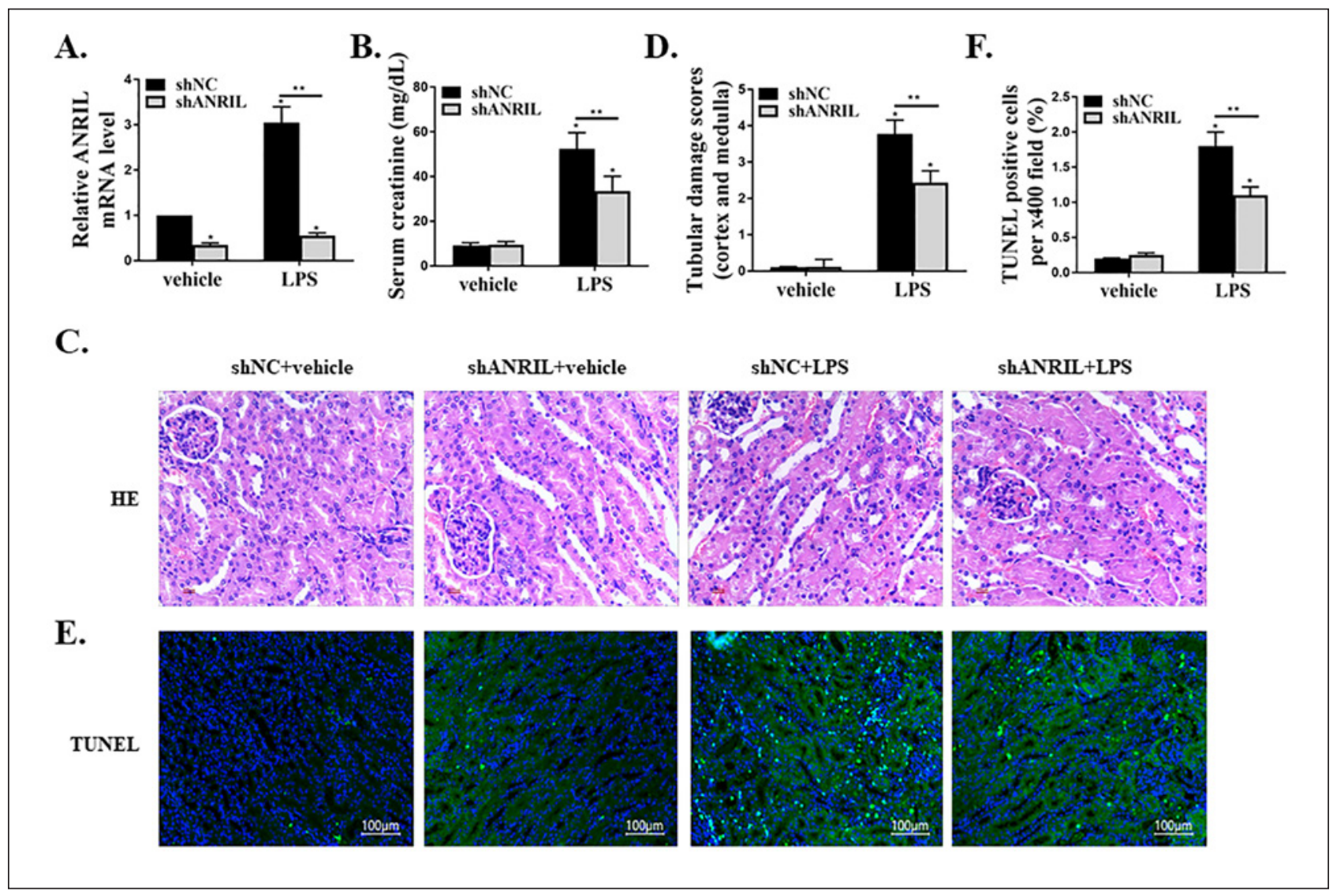

Fig. 2. Downregulated ANRIL expression attenuated LPS-induced renal injury in mice. A Expression of ANRIL mRNA in kidney tissue. B Scr level. C BUN level. D Tubular injury score of hematoxylin and eosin staining. E, F Images of TUNEL staining and TUNEL-positive cells ${ }^{*} p<0.05,{ }^{* *} p<0.05$ compared with the shNC vector; $(n=6)$. Scr, serum creatinine; LPS, lipopolysaccharide; H\&E, hematoxylin and eosin.

Cambridge, MA, USA), TLR4 (Abeam, Cambridge, MA, USA), and GAPDH (Origene, Rockville, MD, USA).

It was placed overnight in the refrigerator at $4{ }^{\circ} \mathrm{C}$. After that, it was incubated with antirabbit IgG for $2 \mathrm{~h}$ at $37^{\circ} \mathrm{C}$. GAPDH antibody was used as an internal standard. The ECL Western blotting kit was used to detect all the protein bands.

\section{Statistical Method}

SPSS 19.0 software was used to analyze the monitored data. Mean \pm SD was used to represent the data analysis results. $T$ test was carried out on the data analysis results of the 2 groups, and one-way ANOVA was used to analyze the data between groups. LSD test was used in the subsequent analysis. $p<0.05$ showed that the difference was statistically significant.

\section{Results}

The Expression of ANRIL was Increased in LPS-Induced Kidney Injury in vivo

Ten mg/kg LPS was injected into the mice, as shown in Figure 1A, and compared with the control group, the Scr level in the LPS group increased significantly and reached its maximum 
A.
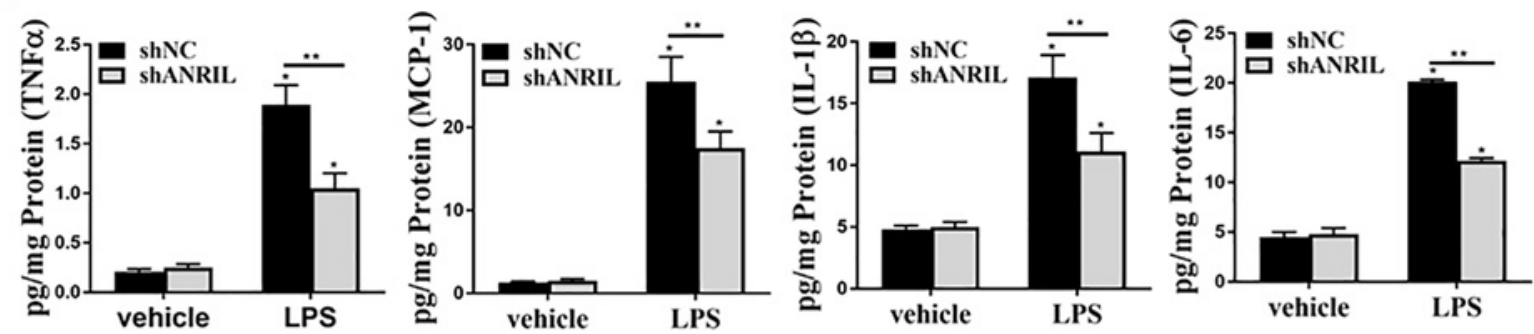

B. shNC+vehicle shANRIL+vehicle
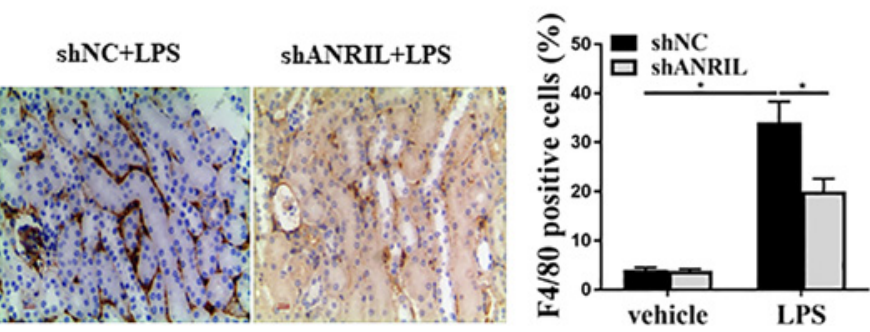

Fig. 3. Down-regulated ANRIL expression inhibited LPS-induced inflammatory responses in mice. A Cytokine levels in mouse serum. B IHC staining of F4/80. ${ }^{*} p<0.05$, ${ }^{* *} p<0.05$ compared with the shNC vector; $(n=6)$. TNF- $\alpha$, tumor necrosis factor alpha; LPS, lipopolysaccharide; IL, interleukin.

in $24 \mathrm{~h}$. According to Figure 1B, serum BUN level in the LPS group was significantly higher than that in the control group, and the serum BUN level in the LPS group reached a peak within $24 \mathrm{~h}$.

As shown in Figure 1C, ANRIL was found to increase at the beginning of $2 \mathrm{~h}$ in the kidneys of the LPS group of mice and reached a peak level at $24 \mathrm{~h}$. As shown in Figure 1D, miR-199a was found to decrease in the kidneys of the LPS group at the beginning of $2 \mathrm{~h}$ and was the lowest at the $24 \mathrm{~h}$ level.

To study the potential mechanism of ANRIL in AKI, ANRIL was into transfected cells. As shown in Figure 1E, the mRNA expression of miR-199a was increased in the si-ANRIL group, and the mRNA expression of miR-199a was reduced in the ANRIL-pcDNA group. It was predicted that miR-199a was a potential target for ANRIL by 3 miRNA databases (TargetScan, PicTar and miRanda; Fig. 1F). WT or MT miR-199a-3'-UTR was cloned to confirm whether ANRIL can directly bind to miR-199a and luciferase activity was assayed. As shown in Figure $1 \mathrm{G}$, overexpression of miRNA-199a mimic can significantly reduce the activity of ANRIL$3^{\prime}$ UTR luciferase $(p<0.01)$, but MT ANRIL-3'UTR luciferase activity has no significant change, which fully indicates that miRNA-199a is the direct target of ANRIL in laryngeal cancer cells.

\section{Downregulated ANRIL Expression Attenuated LPS-Induce Kidney Injuries In vitro}

To analyze the role of ANRIL in LPS-induced AKI, ANRIL expression was knocked down by injecting shANRIL into mice AKI model. As shown in Figure 2A, compared with the control group, the expression of ANRIL in the LPS group decreased significantly, which indicated that the ANRIL knockdown was successful. As shown in Figure 2B, in the LPS group, the Scr level was significantly increased after $24 \mathrm{~h}$ of LPS injection in shNC mice, and the Scr level was significantly decreased in shANRIL mice after LPS injection. The hematoxylin and eosin staining results showed that the kidney damage of the mice was significantly increased $24 \mathrm{~h}$ 

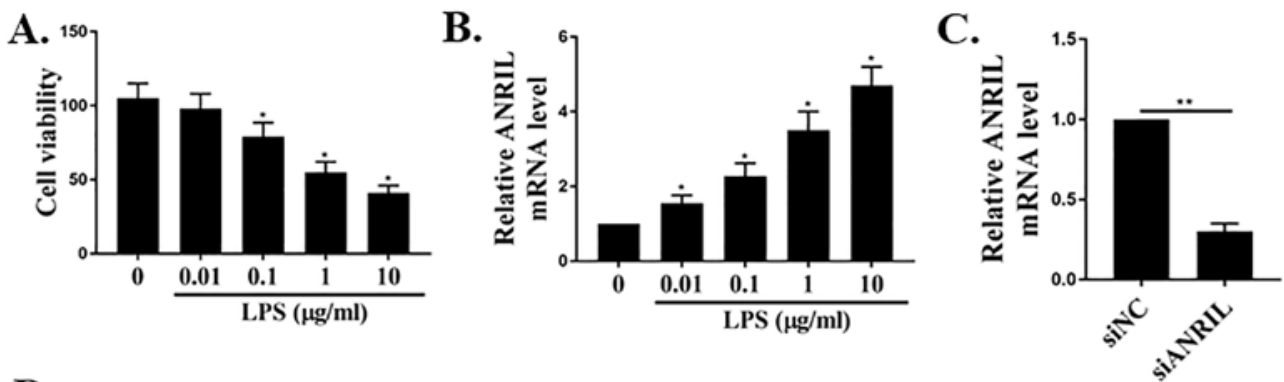

D.
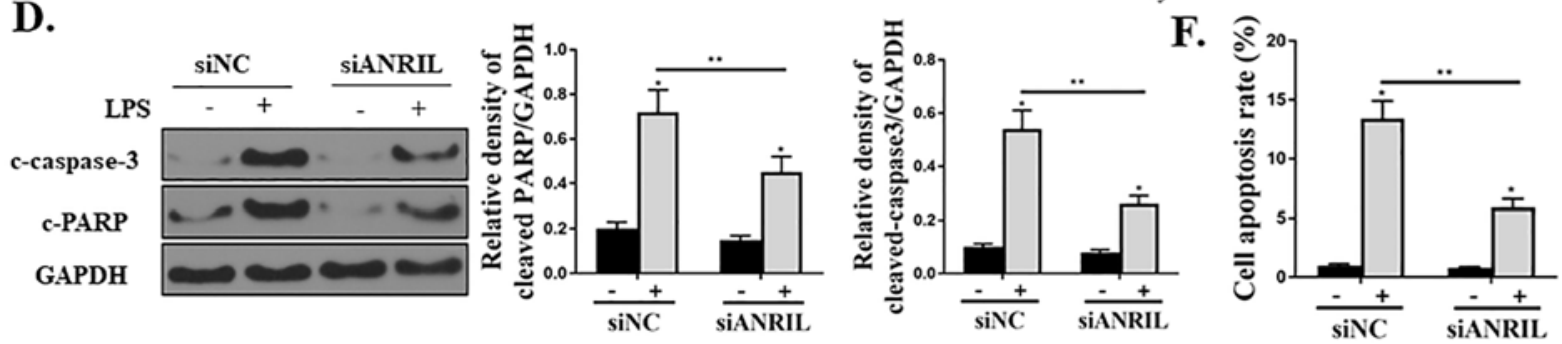

E.
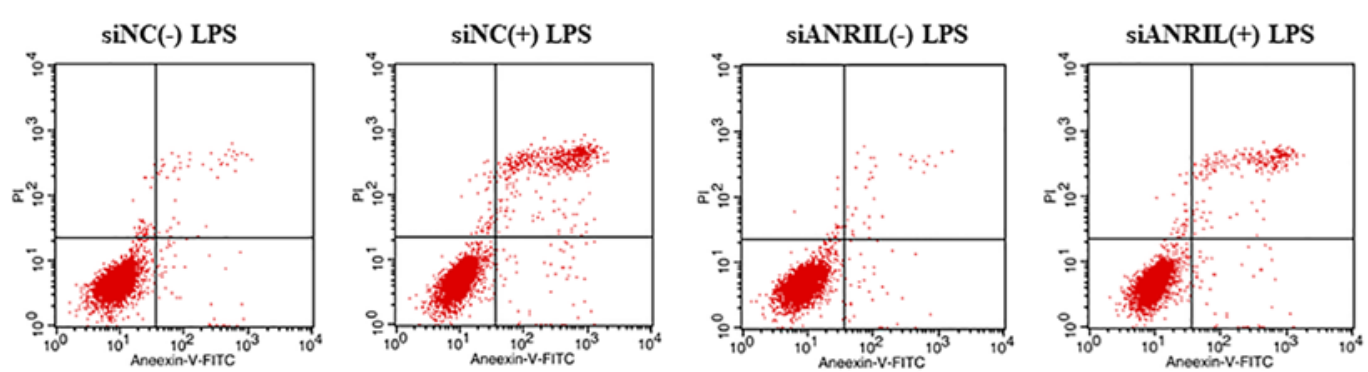

Fig. 4. ANRIL mediated LPS-induced apoptosis in HK-2 cells. A Cell viability. B ANRIL mRNA expression levels. C ANRIL mRNA expression levels in cells transfected with siNC or siANRIL. D Expression levels of cleaved PARP and cleaved caspase-3. E, F Percentage of apoptosis and apoptosis. ${ }^{*} p<0.05,{ }^{* *} p<0.05$ compared with siNC without LPS treatment. LPS, lipopolysaccharide.

after the LPS injection in the shNC mice, and the kidney damage of the shANRIL mice was weakened after the LPS injection (Fig. 2C). Compared with the control group, the tubular lesion scores of the mice were higher after $24 \mathrm{~h}$ of LPS injection in shNC mice, and the shRNAR mice had lower renal tubular injury scores after LPS injection (Fig. 2D). Furthermore, apoptosis was measured by TUNEL staining, from Figure $2 \mathrm{E}$ and F, we can see that compared with the control group, TUNEL-positive cells of the mice increased after $24 \mathrm{~h}$ of LPS injection in shNC mice, and the TUNEL positive cells of shANRIL mice were reduced after LPS injection.

In addition, as shown in Figure 3A, after SHNC mice were injected with LPS for $24 \mathrm{~h}$, inflammatory factors (IL-1 $\beta$, IL-6, MCP-1, and TNF- $\alpha$ ) in vivo were significantly higher than those in the control group. And the levels of inflammatory factors (IL-1 $\beta$, IL-6, MCP-1, and TNF- $\alpha$ ) in shANRIL mice were significantly reduced after LPS injection. In addition, IHC staining showed that kidney neutrophil accumulation increased in mice after $24 \mathrm{~h}$ of LPS injection in shNC mice compared with that in the control group, and renal neutrophil accumulation decreased in shANRIL mice after LPS injection (Fig. 3B). These data suggested that knockdown of ANRIL can inhibit kidney inflammation. 
A.

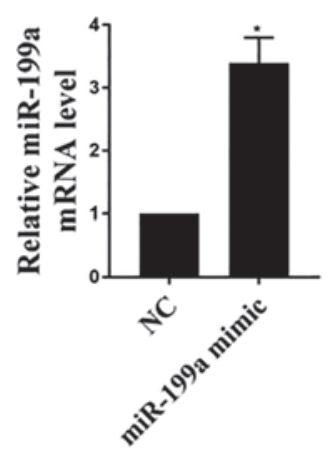

B.

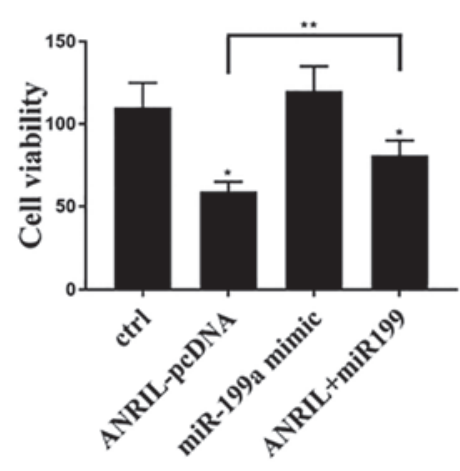

C.

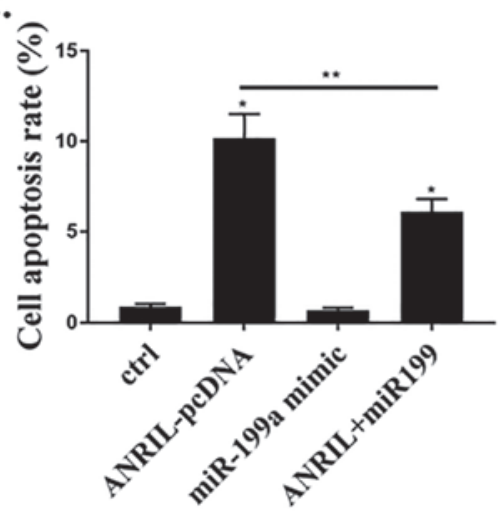

Fig. 5. miR-199a reversed the effect of ANRIL on apoptosis. A miR-199a mRNA levels. B Cell viability assay. C Apoptosis assay.

ANRIL-Mediated LPS-Induced Apoptosis in HK-2 Cells

The role of ANRILin LPS-induced kidney injury was studied. Compared with the control group, the LPS treatment group showed a decrease in cell viability when the treatment time was prolonged (Fig. 4A). As shown in the results of Figure 4B, with the prolongation of the LPS treatment group, the expression of ANRIL in HK-2 cells increased gradually $(p<0.05)$. From Figure 4C, we can see that the expression of ANRIL in the si-ANRIL group was significantly lower than that in the sh-NC group, indicating that ANRIL overexpression was successful. As shown in Figure 4D, the expression of cleaved cystatin-3 and cleaved PARP protein in siNC cells treated with LPS increased significantly, while the expression of cleaved cystatin-3 and cleaved PARP protein in siANRIL cells treated with LPS decreased significantly. From Figure 4E and F, we can see that the apoptotic rate of siNC cells increased after LPS treatment, while that of siANRIL cells decreased after LPS treatment. These data indicated that ANRIL overexpression can inhibit LPS induction.

\section{Effect of miR-199a Reversing ANRIL on Apoptosis}

The effect of ANRIL on apoptosis by targeting miR-199awas further studied. We can know from Figure 5A. Compared with the control group, the expression of mir-199a in the mir-199a simulation group was significantly lower than that in the control group, which fully demonstrated the successful overexpression of mir-199a. As shown in Figure 5B, ANRIL overexpression reduced cell viability, after the addition of microRNA-199a, the effect of ANRIL overexpression on cell viability was reversed. As shown in Figure 5C, ANRIL overexpression induced apoptosis, when mir-199a was added, the effect of ANRIL overexpression on apoptosis was reversed. These data suggest that mir-199a reversed the effect of ANRIL on apoptosis.

ANRIL Activated TLR4/NF-kB Signaling Pathway in LPS-Induced AKI in vivo and in vitro

A recent study found that LPS-induced mouse AKI promoted the development of renal injury by modulating the TLR4/MyD88/NF-kB signaling pathway [18]. To investigate whether ANRIL can regulate LPS-induced renal injury by regulating the LPS-induced renal injury signaling pathway. In vivo results were shown in Figure 6A. The phosphorylation levels of IkBa, NF-kB, and TLR4 in siNCRIL cells treated with LPS were significantly higher than those in the control group. The expression levels of TLR4 phosphorylated NF-kB p65 and phosphorylated IkBa in si-ANRIL cells treated with LPS were significantly decreased. In vitro 


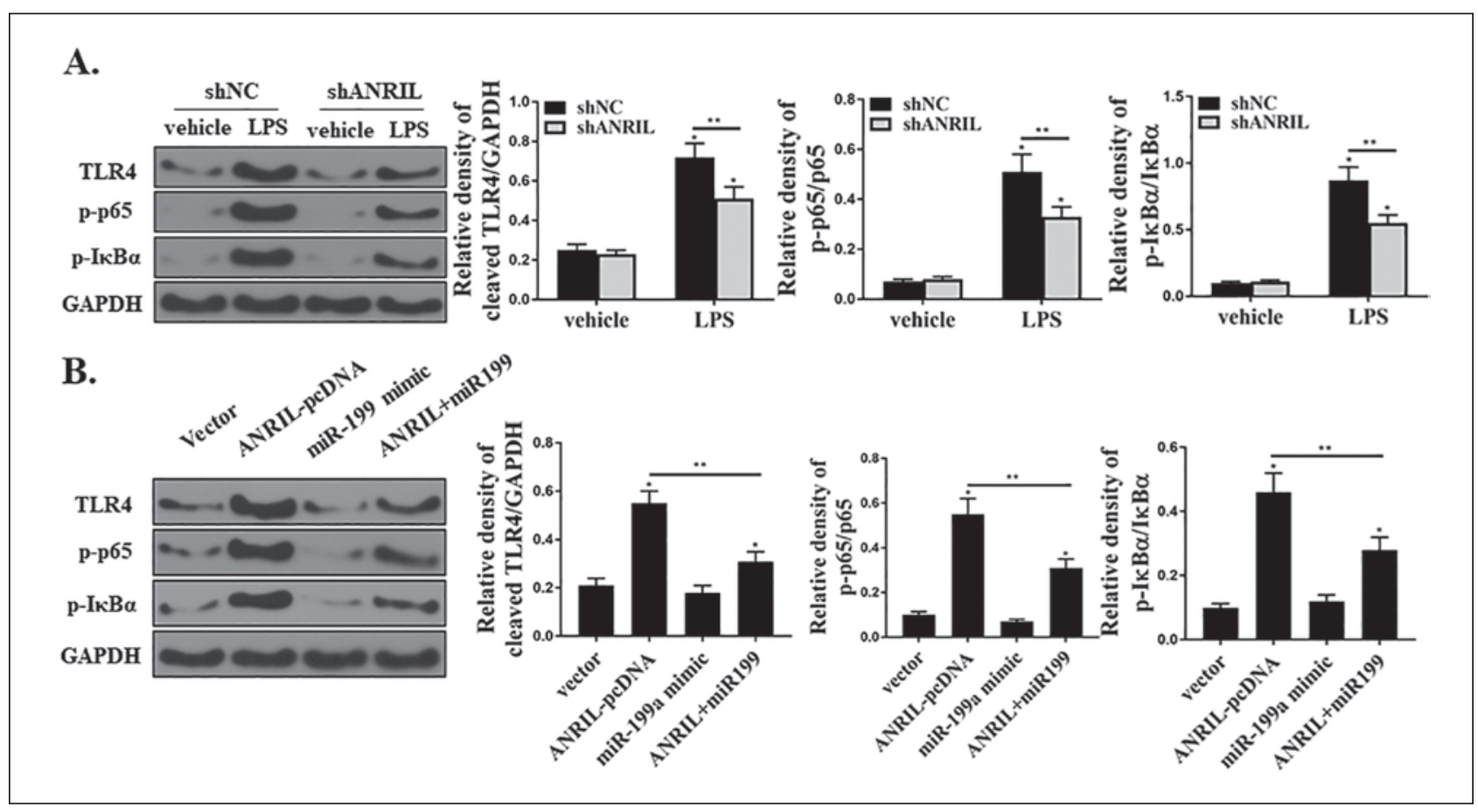

Fig. 6. ANRIL activated the TLR4/Nfkb signaling pathway in LKI induced by LPS. A The expression of TLR4, p-p65 and p-IkBa protein in injections of shNC and shANRIL mice with or without LPS (10 mg/kg). B Expression of p-ikba, p-p65, and TLR4 proteins in hk-2 cells or vectors transfected with Anril-PCDNA. LPS, lipopolysaccharide.

results further confirmed that overexpression of ANRIL in HK-2 cells upregulated TLR4, phosphorylated NF-kB p65, and phosphorylated IkBa expression, while overexpression of miR-199 in HK-2 cells inhibited TLR4, phosphoric acid NF-kB p65, and phosphorylated IkBa expression. The addition of ANRIL reversed the effect of miR-199 overexpression on TLR4, phosphorylated NF-kB p65, and phosphorylated IkBa expression (Fig. 6B).

\section{Role of NF-kB Pathway in ANRIL-Induced Apoptosis}

The results were shown in Figure 7A. The expression levels of p-p65, c-caspase-3, and c-PARP in the ANRIL overexpression group were significantly higher than those in the control group, and the addition of PDTC inhibitor reduced the effect of ANRIL overexpression on p-p65, c-caspase-3, and c-PARP protein expression levels. As shown in Figure 7B, the apoptotic rate of the ANRIL overexpression group was significantly increased compared with the control group, and the addition of the PDTC inhibitor reduced the effect of ANRIL overexpression on the apoptosis rate. These results indicated that ANRIL was capable of biologically acting on AKI through the NF-kB pathway.

\section{Discussion}

For sepsis, the infection causes an uncontrolled systemic inflammatory response, which is excessively released by various inflammatory mediators, with extensive self-tissue and cell damage and organ dysfunction $[19,20]$. Recently, studies have been conducted to identify the pathophysiological mechanisms of acute kidney injury caused by sepsis $[21,22]$. TNF- $\alpha$, IL-1 $\beta$ 
A.
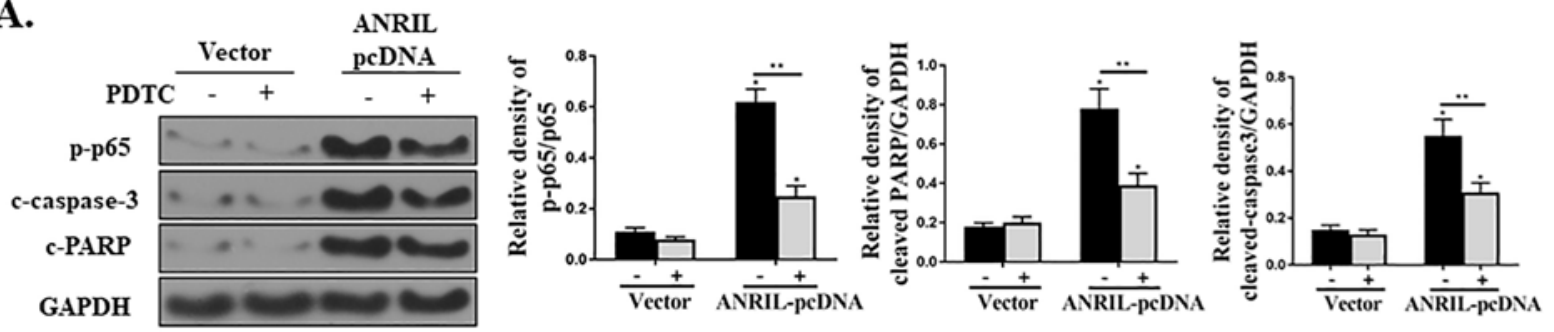

B.
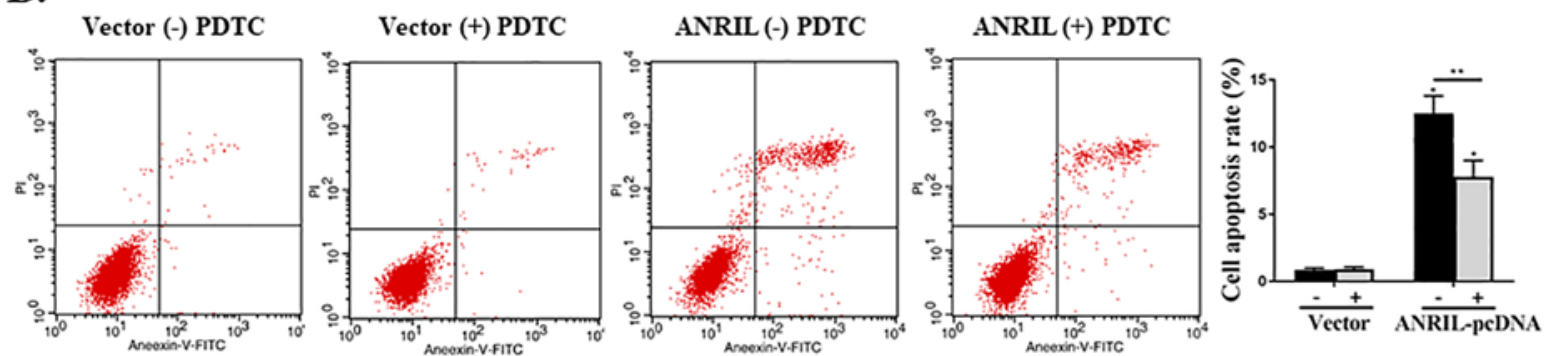

Fig. 7. Role of the NF-kB pathway in ANRIL-induced apoptosis. A p-p65, cleaved PARP and cleaved caspase-3 protein expression levels measured in HK-2 cells. B Apoptosis rate measured in HK-2 cells.

are important proinflammatory factors, which promote the production of cytokine storms and cascade the inflammatory response. These inflammatory mechanisms have also been shown to be involved in the process of acute kidney injury in sepsis and are the main causative factors [23]. This study also confirmed the high release of inflammatory factors TNF- $\alpha$ and IL-1 $\beta$ in the early stage after sepsis modeling.

Previous research found IncRNA plays a very powerful role in gene transcription and posttranscription regulation [24, 25]. LncRNA is abnormally expressed in inflammatory diseases and involved in the activation of various inflammation-related genes and activation of signaling pathways [26]. Previous studies have shown that lncRNA MALAT1 can inhibit NF- $\kappa B$ DNA binding activity, reduce the number of inflammatory cytokines, and weaken the autoimmune inflammatory response. Other studies found that ANRIL can inhibit the expression of inflammatory cytokines to some extent. This study found that the expression of ANRIL in the kidney of LKI-induced AKI mice increased gradually, indicating that ANRIL was involved in LPS-induced acute kidney injury in AKI mice.

BUN and Scr in serum are commonly used biochemical indicators for detecting renal function, and their levels in serum mainly reflect the filtering function of glomeruli [27]. In the pathological changes of renal tissue, the shANRIL group had renal tissue protection compared with the model group, which reduced the release of TUNEL-positive cells and cytokines and the accumulation of neutrophils in the kidneys of mice. In vitro results further indicated that ANRIL overexpression inhibited LPS-induced apoptosis and reduced cleaved caspase-3 expression and cleaved PARP protein levels. These data indicated that ANRIL was involved in the inflammatory response, and ANRIL silencing may partially antagonize the resulting inflammatory response, further protecting the kidney.

miRNAs can target a large number of protein-coding genes as well as lncRNAs [28, 29]. Studies have found that miRNAs are associated with inflammatory diseases [30, 31]. Overex- 
pression of MALAT1 has been found to reduce the inhibitory effect of miR-146 on LPS-induced inflammation. In addition, the knockout of MALAT1 can inhibit inflammation by upregulating miR-146 in LPS-induced acute lung injury [32]. It has been found that upregulation of mir-29b expression in myocardial cells by gene transfection can antagonize renal injury, while lncRNA ANRIL can act as an "endogenous sponge" of miR-29b to downregulate the expression of mir-29b and cause renal injury in mice [33]. We screened miR-199a as a target gene for ANRIL by the database. And ANRIL regulated its expression by targeting the 3 'UTR of the miR-199a gene.

Studies have shown that the TLR4 receptor mediates the activation of NF- $\kappa \mathrm{B}$ in endothelial cells by interleukin receptor homologous domain, interleukin downstream signal transduction molecule MyD88, and TNF receptor-related factors, thus producing immuneinflammatory cytokines, leading to inflammation [34, 35]. Studies have found that MIAT can promote the expression of TLR4 by upregulating miR-93 [36]. The strong expression of tlr4 partially reverses the protective effect of miR-93 overexpression on cardiac hypertrophy. It was found that the silencing of ANRIL reduced LPS-induced TLR4, phosphorylation of NF-kB p65, and phosphorylated IkBa expression, whereas overexpression of ANRIL increased TLR4, phosphorylated NF-kB p65, and phosphorylated IkBa expression. In addition, PDTC inhibitors were capable of inhibiting apoptosis induced by overexpression of ANRIL. It was suggested that ANRIL may inhibit LPS-induced mouse kidney injury through an inflammatory pathway acting on TLR4/MyD88/NF-kB. In the future, we will further study the miR-199a-targeted binding protein and further verify whether the protein plays a regulatory role through TLR4/NF-kB pathway.

\section{Conclusion}

ANRIL can protect against LPS-induced acute kidney injury in mice by regulating mir-199a and NF-kB p65, suggesting that ANRIL may be a potential therapeutic target for AKI, this will lay a solid theoretical foundation for the clinical treatment of AKI by ANRIL.

\section{Acknowledgments}

Not applicable.

\section{Statement of Ethics}

The animal work was taken place at The Fifth Affiliated Hospital of Sun Yat-sen University and experimental scheme was approved by the Animal Protection and Use Ethics Committee of The Fifth Affiliated Hospital of Sun Yat-sen University.

\section{Disclosure Statement}

The authors declare that they have no competing interests. 
Kidney
Blood Pressure
Research

\begin{tabular}{l|l}
\hline Kidney Blood Press Res 2020;45:209-221 \\
\hline DOI: 10.1159/000505154 & $\begin{array}{l}\text { @ 2020 The Author(s). Published by S. Karger AG, Basel } \\
\text { www.karger.com/kbr }\end{array}$ \\
\hline
\end{tabular}

Zhu et al.: Long Noncoding RNA ANRIL and Kidney Injury

\section{Funding Sources}

This study was supported in part by grants of the National Natural Science Foundation of China (NSFC no. 81600529), the Fundamental Research Funds for the Central Universities (17ykpy64), and Science and Technology Program of Zhuhai, China (20181117E030069).

\section{Availability of Data and Materials}

The analyzed data sets generated during the study are available from the corresponding author on reasonable request.

\section{Author Contribution}

Y.Z. and S.-W.W.: study design, experiments, data analysis, manuscript preparation. A.D., W.-P.Z., M.-F.M., T.-X.C., H.Y., and H.Z.: experiments, data analysis, manuscript preparation.

\section{References}

1 Rhodes A, Evans LE, Alhazzani W, Levy MM, Antonelli M, Ferrer R, et al. Surviving Sepsis Campaign: International Guidelines for Management of Sepsis and Septic Shock: 2016. Crit Care Med. 2017 Mar;45(3):486-552.

2 Alberti C, Brun-Buisson C, Burchardi H, Martin C, Goodman S, Artigas A, et al. Epidemiology of sepsis and infection in ICU patients from an international multicentre cohort study. Intensive Care Med. 2002 Feb;28(2): 108-21.

3 Wieczorek A, Tokarz A, Gaszynski W, Gaszynski T. The doripenem serum concentrations in intensive care patients suffering from acute kidney injury, sepsis, and multi organ dysfunction syndrome undergoing continuous renal replacement therapy slow low-efficiency dialysis. Drug Des Devel Ther. 2014 Oct;8:203944.

4 Lentini P, de Cal M, Clementi A, D’Angelo A, Ronco C. Sepsis and AKI in ICU Patients: The Role of Plasma Biomarkers. Crit Care Res Pract. 2012;2012:856401.

5 Godin M, Murray P, Mehta RL. Clinical approach to the patient with AKI and sepsis. Semin Nephrol. 2015 Jan; 35(1):12-22.

6 Bishop BM, Bon JJ, Trienski TL, Pasquale TR, Martin BR, File TM Jr. Effect of introducing procalcitonin on antimicrobial therapy duration in patients with sepsis and/or pneumonia in the intensive care unit. Ann Pharmacother. 2014 May;48(5):577-83.

7 Walke T, Chalfin D, Lee J, Rivers E. Cost-effectiveness of a rapid and accurate test for diagnosing infection in severe sepsis and septic shock patients. Crit Care. 2010;14 Suppl 1:P48.

8 Fukazawa K, Lee HT. Volatile anesthetics and AKI: risks, mechanisms, and a potential therapeutic window. J Am Soc Nephrol. 2014 May;25(5):884-92.

9 Agarwal A, Dong Z, Harris R, Murray P, Parikh SM, Rosner MH, et al.; Acute Dialysis Quality Initiative XIII Working Group. Cellular and Molecular Mechanisms of AKI. J Am Soc Nephrol. 2016 May;27(5):1288-99.

10 Ratliff BB, Rabadi MM, Vasko R, Yasuda K, Goligorsky MS. Messengers without borders: mediators of systemic inflammatory response in AKI. J Am Soc Nephrol. 2013 Mar;24(4):529-36.

11 Van der Auwera I, Limame R, van Dam P, Vermeulen PB, Dirix LY, Van Laere SJ. Integrated miRNA and mRNA expression profiling of the inflammatory breast cancer subtype. Br J Cancer. 2010 Aug;103(4):532-41.

12 Schouten M, Wiersinga WJ, Levi M, van der Poll T. Inflammation, endothelium, and coagulation in sepsis. J Leukoc Biol. 2008 Mar;83(3):536-45.

13 Yang L, Lin C, Jin C, Yang JC, Tanasa B, Li W, et al. IncRNA-dependent mechanisms of androgen-receptor-regulated gene activation programs. Nature. 2013 Aug;500(7464):598-602.

14 Zou ZW, Ma C, Medoro L, Chen L, Wang B, Gupta R, et al. LncRNA ANRIL is up-regulated in nasopharyngeal carcinoma and promotes the cancer progression via increasing proliferation, reprograming cell glucose metabolism and inducing side-population stem-like cancer cells. Oncotarget. 2016 Sep;7 (38):61741-54.

15 Wang Y, Cheng N, Luo J. Down-regulation of IncRNA ANRIL represses tumorigenicity and enhances cisplatininduced cytotoxicity via regulating microRNA let-7a in nasopharyngeal carcinoma. J Biochem Mol Toxicol. 2017;31(7):e21904.

16 Cao Y, Xu R, Xu X, Zhou Y, Cui L, He X. Downregulation of IncRNA CASC2 by microRNA-21 increases the proliferation and migration of renal cell carcinoma cells. Mol Med Rep. 2016 Jul;14(1):1019-25. 
17 Butchart LC, Fox A, Shavlakadze T, Grounds MD. The long and short of non-coding RNAs during post-natal growth and differentiation of skeletal muscles: focus on IncRNA and miRNAs. Differentiation. 2016 Dec;92(5): 237-48.

18 Zhou ZQ, Wang ZK, Zhang L, Ren YQ, Ma ZW, Zhao N, et al. Role of ESAT-6 in renal injury by regulating microRNA-155 expression via TLR4/MyD88 signaling pathway in mice with Mycobacterium tuberculosis infection. Biosci Rep. 2017 Jul;37(4):BSR20170021.

19 Hotchkiss RS, Nicholson DW. Apoptosis and caspases regulate death and inflammation in sepsis. Nat Rev Immunol. 2006 Nov;6(11):813-22.

20 Schouten M, Wiersinga WJ, Levi M, Tom VDP. Inflammation, endothelium, and coagulation in sepsis. J Leukoc Biol. 2008 Mar;83(3):536-45.

21 Souza AC, Yuen PS, Star RA. Microparticles: markers and mediators of sepsis-induced microvascular dysfunction, immunosuppression, and AKI. Kidney Int. 2015 Jun;87(6):1100-8.

22 Xu X, Wang J, Yang R, Dong Z, Zhang D. Genetic or pharmacologic inhibition of EGFR ameliorates sepsisinduced AKI. Oncotarget. 2017 Sep;8(53):91577-92.

23 Barber RC, Aragaki CC, Rivera-Chavez FA, Purdue GF, Hunt JL, Horton JW. TLR4 and TNF- $\alpha$ polymorphisms are associated with an increased risk for severe sepsis following burn injury. J Med Genet. 2004 Nov;41(11): 808-13.

24 Yang G, Lu X, Yuan L. LncRNA: a link between RNA and cancer. Biochim Biophys Acta. 2014 Nov;1839(11): 1097-109.

25 Chen G, Wang Z, Wang D, Qiu C, Liu M, Chen X, et al. LncRNADisease: a database for long-non-coding RNAassociated diseases. Nucleic Acids Res. 2013 Jan;41(Database issue):D983-6.

26 Wang H, Cao Q, Ge J, Liu C, Ma Y, Meng Y, et al. LncRNA-regulated infection and inflammation pathways associated with pregnancy loss: genome wide differential expression of IncRNAs in early spontaneous abortion. Am J Reprod Immunol. 2014 Oct;72(4):359-75.

27 Vaidya VS, Ozer JS, Dieterle F, Collings FB, Ramirez V, Troth S, et al. Kidney injury molecule-1 outperforms traditional biomarkers of kidney injury in preclinical biomarker qualification studies. Nat Biotechnol. 2010 May;28(5):478-85.

28 Ballantyne MD, McDonald RA, Baker AH. IncRNA/MicroRNA interactions in the vasculature. Clin Pharmacol Ther. 2016 May; 99(5):494-501.

29 Fu N, Zhao SX, Kong LB, Du JH, Ren WG, Han F, et al. LncRNA-ATB/microRNA-200a/ $\beta$-catenin regulatory axis involved in the progression of HCV-related hepatic fibrosis. Gene. 2017 Jun;618:1-7.

30 O'Connell RM, Kahn D, Gibson WS, Round JL, Scholz RL, Chaudhuri AA, et al. MicroRNA-155 promotes autoimmune inflammation by enhancing inflammatory T cell development. Immunity. 2010 0ct;33(4):607-19.

31 Kurowska-Stolarska M, Alivernini S, Ballantine LE, Asquith DL, Millar NL, Gilchrist DS, et al. MicroRNA-155 as a proinflammatory regulator in clinical and experimental arthritis. Proc Natl Acad Sci USA. 2011 Jul;108(27): 11193-8.

32 Dai L, Zhang G, Cheng Z, Wang X, Jia L, Jing X, et al. Knockdown of LncRNA MALAT1 contributes to the suppression of inflammatory responses by up-regulating miR-146a in LPS-induced acute lung injury. Connect Tissue Res. 2018 Nov;59(6):581-92.

33 Sun SF, Tang PM, Feng M, Xiao J, Huang XR, Li P, et al. Novel IncRNA Erbb4-IR Promotes Diabetic Kidney Injury in db/db Mice by Targeting miR-29b. Diabetes. 2018 Apr;67(4):731-44.

34 Menghini R, Campia U, Tesauro M, Marino A, Rovella V, Rodia G, et al. Toll-like receptor 4 mediates endothelial cell activation through NF- $\kappa B$ but is not associated with endothelial dysfunction in patients with rheumatoid arthritis. PLoS One. 2014 Jun; 9(6):e99053.

35 Ni H, Jin W, Zhu T, Wang J, Yuan B, Jiang J, et al. Curcumin modulates TLR4/NF- $\mathrm{kB}$ inflammatory signaling pathway following traumatic spinal cord injury in rats. J Spinal Cord Med. 2015 Mar;38(2):199-206.

36 Li Y, Wang J, Sun L, Zhu S. LncRNA myocardial infarction-associated transcript (MIAT) contributed to cardiac hypertrophy by regulating TLR4 via miR-93. Eur J Pharmacol. 2018 Jan;818:508-17. 